\title{
A systematic review of taeniasis, cysticercosis and trichinellosis in Vietnam
}

Dinh Ng-Nguyen ${ }^{1,2^{*}}$, Mark A. Stevenson ${ }^{1}$ and Rebecca J. Traub ${ }^{1}$

\begin{abstract}
Taeniasis, cysticercosis and trichinellosis have been ranked as the most important food-borne parasites of humans in terms of public health, socioeconomic and trade impact. Despite this, information on these food-borne zoonoses in Vietnam is scarce and fragmented, and many local reports remain inaccessible to the international research community. This study aims to conduct comprehensive literature searches to report on the incidence and estimate the true prevalence of taeniasis in humans and T. solium cysticercosis in humans and pigs in Vietnam utilizing Bayesian models; in addition, to report the incidence and the distribution of trichinellosis. A Bayesian approach was used to estimate the true prevalence of taeniasis and cysticercosis based on published diagnostic test characteristics used in each published cross-sectional survey. The utilization of coproscopic-based examination of Taenia eggs in stool, although highly specific for genus-level detection, has poor sensitivity and led to an underestimation of the prevalence of human taeniasis. Similarly, post-mortem-based surveys of T. solium cysticercosis in pigs also led to the underestimation of prevalence of porcine cysticercosis. On the other hand, the low specificity of immunodiagnostic methods, in particular Ab-ELISA, led to a likely overestimation of $T$. solium cysticercosis in humans. Due to the use of imperfect diagnosis tests combined with poor descriptions of sampling methods, our ability to draw solid conclusions from these data is limited. We estimate that the true prevalence of taeniasis and T. solium cysticercosis in rural 'hotspots', is as high as 13\% for each, in humans. Taeniasis and T. solium cysticercosis occurs in 60 of the 63 provinces of Vietnam. Most of the information relating to the distribution and prevalence of porcine cysticercosis is limited to commercial abattoir surveys. In Vietnam, Taenia asiatica appears to be confined to the north where it occurs sympatrically with $T$. solium and Taenia saginata. The status of T. asiatica in Central and South Vietnam remains unascertained. To date, five outbreaks of trichinellosis have been reported in the north and northwest of Vietnam, affecting a total of 114 people and responsible for eight fatalities. In the same region, studies of free-roaming pigs showed evidence of high levels of exposure to Trichinella and, in cases where larvae were recovered, the species present were identified as Trichinella spiralis. Based on five studies, the main risk factors for pork-borne zoonoses in Vietnam include the consumption of undercooked/raw meat and vegetables and the use of night-soil for fertilization of local produce. This systematic review draws attention to the importance of these pork-borne zoonoses.
\end{abstract}

Keywords: Cysticercosis, Taeniasis, Trichinellosis, Food-borne zoonoses, Bayesian model, Vietnam

\footnotetext{
*Correspondence: ngocn4@student.unimelb.edu.au; theeveret@gmail.com

${ }^{1}$ Faculty of Veterinary and Agricultural Sciences, University of Melbourne,

Parkville, VIC 3052, Australia

${ }^{2}$ Faculty of Animal Sciences and Veterinary Medicine, Tay Nguyen University,

Dak Lak province, Vietnam
} 


\section{Background}

Taeniasis, cysticercosis and trichinellosis have been ranked as the most important food-borne parasites of humans in terms of public health, socioeconomic and trade impact [1]. In 2010 it was estimated that approximately 300,000 individuals were infected with $T$. solium cysticercosis globally, resulting in over 28,000 deaths [2]. Between 2.5 and 5 million people are estimated to harbour adult tapeworms of T. solium [3-5]. Trichinellosis is reported to be present in 55 countries [6] with 66,000 individuals estimated to have been infected during the period 1986 to 2009 [1]. In 2010 it was estimated that globally there were around 4400 cases of trichinellosis reported, with four deaths [2]. Humans act as the definitive hosts for all three-tapeworm species (Taenia solium, Taenia saginata and Taenia asiatica) via the ingestion of undercooked/raw meat and/or offal [7-9]. Swine are the intermediate hosts of $T$. solium and $T$. asiatica whereas cattle are the intermediate host for $T$. saginata [10]. Humans may also become infected with cysticerci of $T$. solium. In humans, the symptoms of taeniasis are subtle and mild and include abdominal distension, abdominal pain, digestive disorders and anal pruritis $[8,11]$. The signs of T. solium neurocysticercosis (NCC) in humans, on the other hand, are distinctive and include seizures, paralysis, dementia, chronic headache, blindness or even death $[12,13]$.

Parasites of the genus Trichinella comprise nine species and four genotypes divided into two groups, encapsulated and non-encapsulated [14]. There are two epidemiological cycles of Trichinella: domestic and sylvatic. The domestic cycle occurs between domestic animals, particularly domestic pigs and rodents. Of all Trichinella species and genotypes, $T$. spiralis is the most widely distributed and most adapted to domestic pigs, accounting for the most cases of human trichinellosis in Asia, however other sylvatic species such as T. pseudospiralis and T. papuae have also been associated with human outbreaks in the region $[15,16]$. Typically, trichinellosis results in nausea, diarrhoea, high fever, petechial and nailbed hemorrhages, periorbital oedema and myalgia [17]. The severity of symptoms in humans are directly proportional to the number of larvae ingested.

Taeniasis, T. solium cysticercosis [18] and trichinellosis [6] have been reported as endemic in Southeast Asia, including Vietnam. Vietnam has a population of around 90 million people that reside in 63 provinces. There are 54 ethnic groups and approximately $67 \%$ of the population live in rural areas $[19,20]$. The standard of living in most rural communities is poor. Open defecation using outdoor latrines is common practice and livestock access to these latrine areas is, for the most part, unrestricted. Vietnam is grouped as a lower middle-income country with a Gross Domestic Product (GDP) per capita of
USD 2052, ranking it 116th out of 188 countries listed in the United Nations Human Development Index [21, 22]. There are only 79 physicians per 100,000 people; however, this figure is likely to be much lower in remote regions [20]. Moreover, consumption of raw or undercooked blood, meat and organs from domesticated and wild pigs and cattle and raw vegetables is common practice, as are the practices of using night-soil and wastewater to fertilize and irrigate crops $[23,24]$. These factors combined, are highly conducive for the transmission of taeniasis, $T$. solium cysticercosis and trichinellosis.

Vietnam's domestic livestock sector comprises approximately 8 million cattle and buffalo, and 27 million pigs, which produce nearly 3800 million tons of meat products annually [20]. Two primary types of pig- and cattle-husbandry practices exist: commercial farming and backyard husbandry. In rural regions, backyard husbandry practices dominate [23], although the trend is rapidly changing to expand to commercial farming practices aimed at increasing productivity by using hybrid rather than local/traditional breeds. The local/traditional breeds are, however, still raised in many remote and rural areas to use as a supply of food for their owners or nearby communities [25]. The practice of nonconfinement of pigs and cattle is common in rural regions [25] with slaughter activities commonly carried out in backyards without official meat inspection. Meat inspection is only carried out at abattoirs or slaughterpoints, which operate at the district level and/or clusters of large villages.

This review provides updated literature on the incidence and distribution, and critically evaluates both available and previously unavailable local reports on taeniasis, $T$. solium cysticercosis and trichinellosis in Vietnam. As such, it aims to inform the distribution of reported cases of trichinellosis, and estimate the true prevalence (TP) of taeniasis in humans, and T. solium cysticercosis in humans and pigs in Vietnam utilizing Bayesian models thus drawing attention to the importance of these pork-borne zoonoses.

\section{Methods}

We selected published articles reporting on the prevalence, incidence and/or occurrence of Taenia and Trichinella in humans and pigs in Vietnam. The results of the search were not restricted by time, journal, or status of publication. The protocol of this systematic review followed guidelines of the Preferred Reporting Items for Systematic reviews and Meta-Analyses (PRISMA) [26] (see Additional file 1).

\section{Search protocol}

Information from published articles for this study was sourced in two ways: (i) through international and 
Vietnamese online database searches, (ii) through Vietnamese publications obtained from hospitals, government, and research institution and university libraries. Online database searches were performed using PubMed, CABI abstract, Web of Science, MEDLINE (Web of Knowledge) and Scopus. English search terms and keywords in the Boolean operators were used as follows: (Taenia solium OR Taenia saginata OR Taenia asiatica OR human tapeworms OR cysticercosis OR neurocysticercosis OR taeniasis OR taeniosis OR Trichinella OR trichinellosis) AND (epidemiology OR prevalence) AND (human OR porcine OR swine OR pig OR bovine OR cattle OR cow OR calf) AND Vietnam. Scientific names and Vietnamese synonym names of Taenia and Trichinella were used to search databases of the National Library of Vietnam (NLV) and the National Agency for Science and Technology Information of Vietnam (NASATI, http://db.vista.gov.vn). Published articles written in Vietnamese were also sourced through the public search engine (Google.com.vn) using Vietnamese keywords for Taenia or Trichinella.

\section{Study selection}

After independent identification, duplicate articles were removed. The titles and abstracts of articles were then critically evaluated and excluded in cases where they did not address the prevalence, and/or incidence of taeniasis, T. solium cysticercosis and/or Trichinella in humans and/or pigs. Full-text records were excluded if they were review articles; or two articles using the same data; or were not written in English or Vietnamese; or not addressing Taenia and/or Trichinella in humans and pigs in Vietnam. Remaining full-text articles were included for this review. Risk of bias was reduced by only including published reports and excluding studies in which sampling was not comprehensive, such as: source of samples not identified, or where the sample size was not clear for inclusion in the Bayesian modelling (see Statistical analysis).

\section{Data collection}

The following data were retrieved from each study: the title, authors, year of publication, time, sample source/ place, sample size, number of positive samples, diagnostic method utilised for prevalence/incidence determination and factors effecting prevalence, incidence and distribution of positively identified samples.

\section{Statistical analysis}

In this review the prevalence of taeniasis and cysticercosis, as documented in each of the selected published articles is reported as 'apparent prevalence', (AP). That is, for a given number of individuals sampled and tested using a given diagnostic method, AP equals the number of test-positive individuals divided by the total number of individuals tested. Given differences in the diagnostic test protocols used in each of the cross-sectional studies reported in this review, AP estimates have been adjusted to TP estimates taking into account imperfect diagnostic sensitivity (Se) and specificity ( $\mathrm{Sp}$ ) using the general approach described by Rogan \& Gladen [27], and modified for the low prevalence situation using Bayesian methods as described by Messam et al. [28].

In brief, the Bayesian approach accounts for uncertainty in the values of TP and diagnostic Se and Sp of the testing protocol. If $x$ equals the number of individuals testing positive using a diagnostic test of Se and Sp, the distribution of the number of test positive individuals is $\boldsymbol{x} \mid(\boldsymbol{T P}, \boldsymbol{S e}, \boldsymbol{S p}) \sim \operatorname{binomial}(\boldsymbol{n}, \boldsymbol{A P})$ where $\boldsymbol{A P}=$ $T P \times S e+(1-T P)(1-S p)$.

To estimate the TP of taeniasis and cysticercosis for each study beta prior distributions for Se and Sp were used. We assumed that the TP of taeniasis and cysticercosis was not equal to zero and that all prevalences were equally likely, which translates to a $\boldsymbol{T P} \sim \boldsymbol{b e t a}(1,1)$ distribution. Markov chain Monte Carlo (MCMC) methods were used to derive posterior estimates of TP using WinBUGS $[29,30]$. In WinBUGS, the MCMC sampler was run for 200,000 iterations and the first 1000 'burn in' samples were discarded. The posterior distribution of TP was obtained by running sufficient iterations to ensure that the Monte Carlo standard error of the posterior means were at least one order of magnitude smaller than their posterior standard deviation [31]. The point estimate and 95\% credible interval (CrI) for TP is reported as the median and 0.025 and 0.975 quantiles of the posterior distribution of TP.

\section{Results}

Records used for quality and quantity analysis

The protocol for selecting the published records is shown in Fig. 1. There were initially 75 records retrieved from online data, the internet and other resources. Of these records, 40 records were eligible for qualitative and quantitative (23 records) analysis. Of 40 retrieved records, 13 studies were published in international journals, 24 studies were published in national journals and three records were circulated within professional institutes. The numbers of record studied on taeniasis, and both taeniasis and human cysticercosis were 12 and 10 , respectively; whereas there were eight papers report on porcine cysticercosis. Research on Trichinella and trichinellosis were identified in seven records, in which three were studies of humans, three were studies of pigs and one was a study of both of humans and pigs. Our systematic search identified only one (Hanoi-based) study reporting on the prevalence of bovine cysticercosis between 2002 and 2003. 


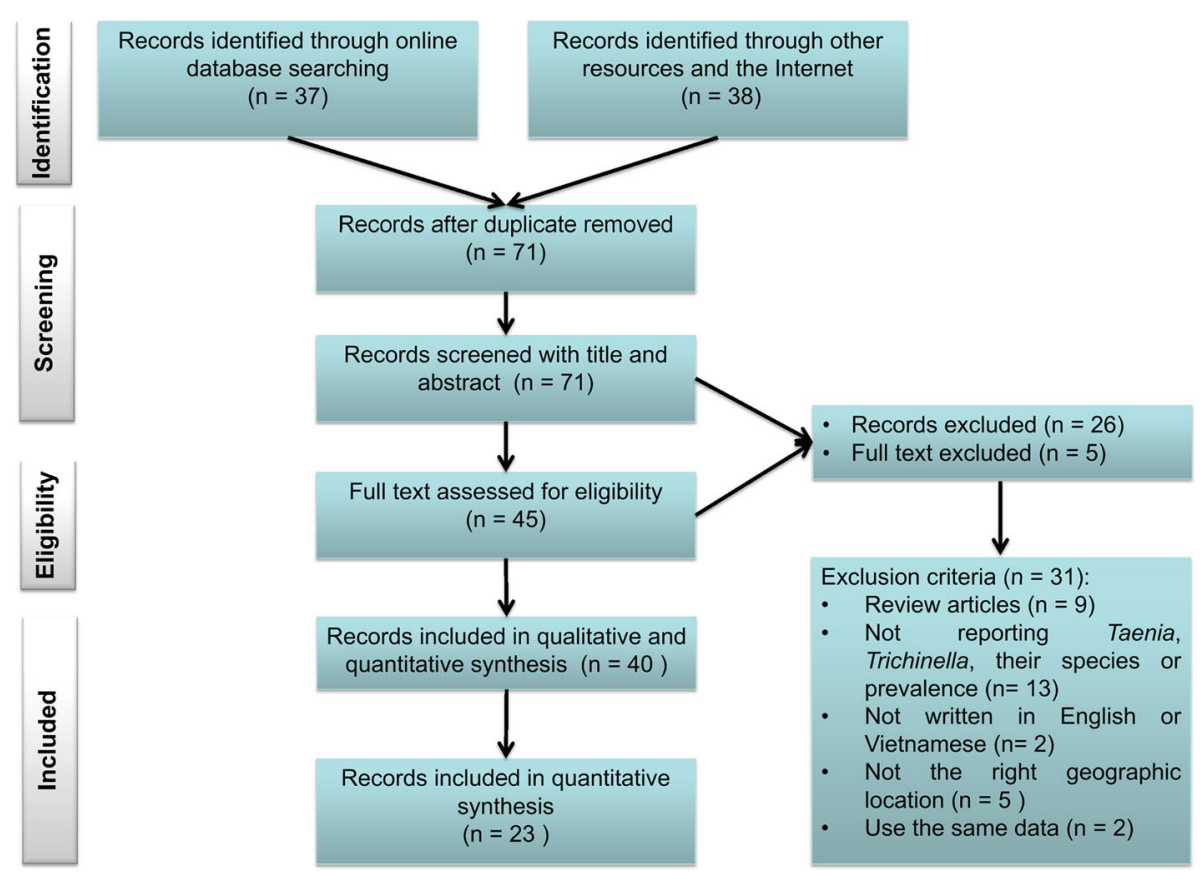

Fig. 1 Flow diagram of searching strategy. Diagram showing the strategy steps of searching and justification for taeniasis, cysticercosis and trichinellosis in Vietnam

Tables 1, 2 and 3 provide a summary of each of the studies cited in this review. For each study the sampling protocol (if known) is reported as well as the diagnostic test used, the number of individuals sampled, the number of individuals testing positive, and the apparent and TP estimates. For both prevalence estimates 95\% CrI for the true population values are reported.

\section{Human taeniasis}

Data on the AP and estimated TP of human taeniasis is summarized in Table 1. Data on the prevalence of taeniasis in Vietnam varied markedly across study sites depending on dietary habits, pig husbandry practices and the socio-economic status of study participants. Human taeniasis was reported in 50 of the 63 provinces of Vietnam [32], with the average province-level AP estimates from $0.11 \%$ in Hoa Binh in the north to $10 \%$ in Kon Tum, in the Central Highlands (Table 1).

In North Vietnam, the AP of taeniasis between 2002 and 2012 ranged from 0.11 to $8.65 \%$ (Table 1). In the North of Vietnam, high foci of taeniasis were found in the provinces of Yen Bai (9.0\%) [33], Bac Ninh (12.68\%) [32] and Phu Tho (8.65\%) [34]. In Ha Giang, located in Northeast Vietnam, 5 out of $84(6.0 \%)$ people were positive for Taenia spp. [35]. In contrast, a retrospective study of 6570 patients presenting to Thai Binh Medical University Hospital with digestive disorders between 2008 and 2010 found that only $11(0.17 \%)$ people were confirmed positive for taeniasis [36].
In 2005, the Institute of Malariology, Parasitology and Entomology Quy Nhon (IMPE-QN) reported $0.33 \%$ of the population to be positive for taeniasis, based on a large-scale helminth survey using the Kato-Katz method. This study involved 35,651 participants in 14 provinces spanning the Central and Central Highland regions of Vietnam [37]. The AP of taeniasis in Chuong and Van Tuan in 2011 and 2014 was relatively high (8\%; 95\% CI: $7-9 \%$ and 10\%; $95 \%$ CI: $8-13 \%$, respectively). It should be noted that sampling in this study was targeted towards those individuals known to habitually consume raw or undercooked beef. Although the sampling protocol used in this study was likely to result in an estimate of the prevalence of taeniasis that was greater than that of the general population, the TP among habitual raw or undercooked beef consumers was 11.0\% (95\% CrI: $7.0-21 \%$ ) and $13.5 \%$ (95\% CrI: $8.0-21 \%$ ) in 2011 and 2014, respectively (Table 1 ). To the best of our knowledge, to date there have been no reports on the prevalence of taeniasis in South Vietnam (Fig. 2).

Consumption of raw and/or undercooked pork or beef in traditional dishes such as 'Nem Chua', undercooked liver, undercooked-beef/pork noodle soup, grilled pork/beef and visceral-raw blood [33, 34, 38] have been identified as major risk factors for taeniasis in the above-mentioned provinces. For all epidemiological studies of taeniasis carried out across Vietnam, it has consistently been shown that males 


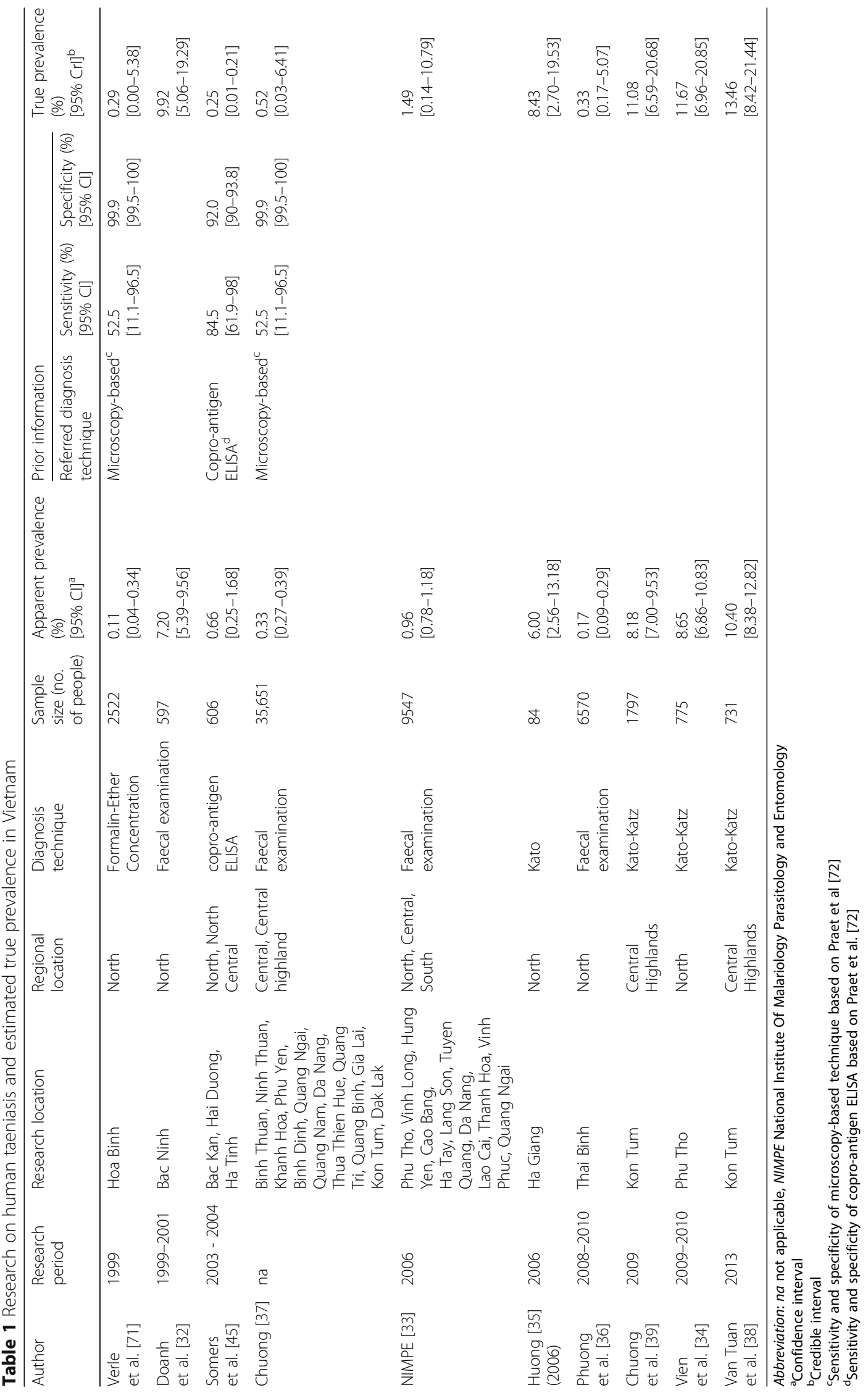




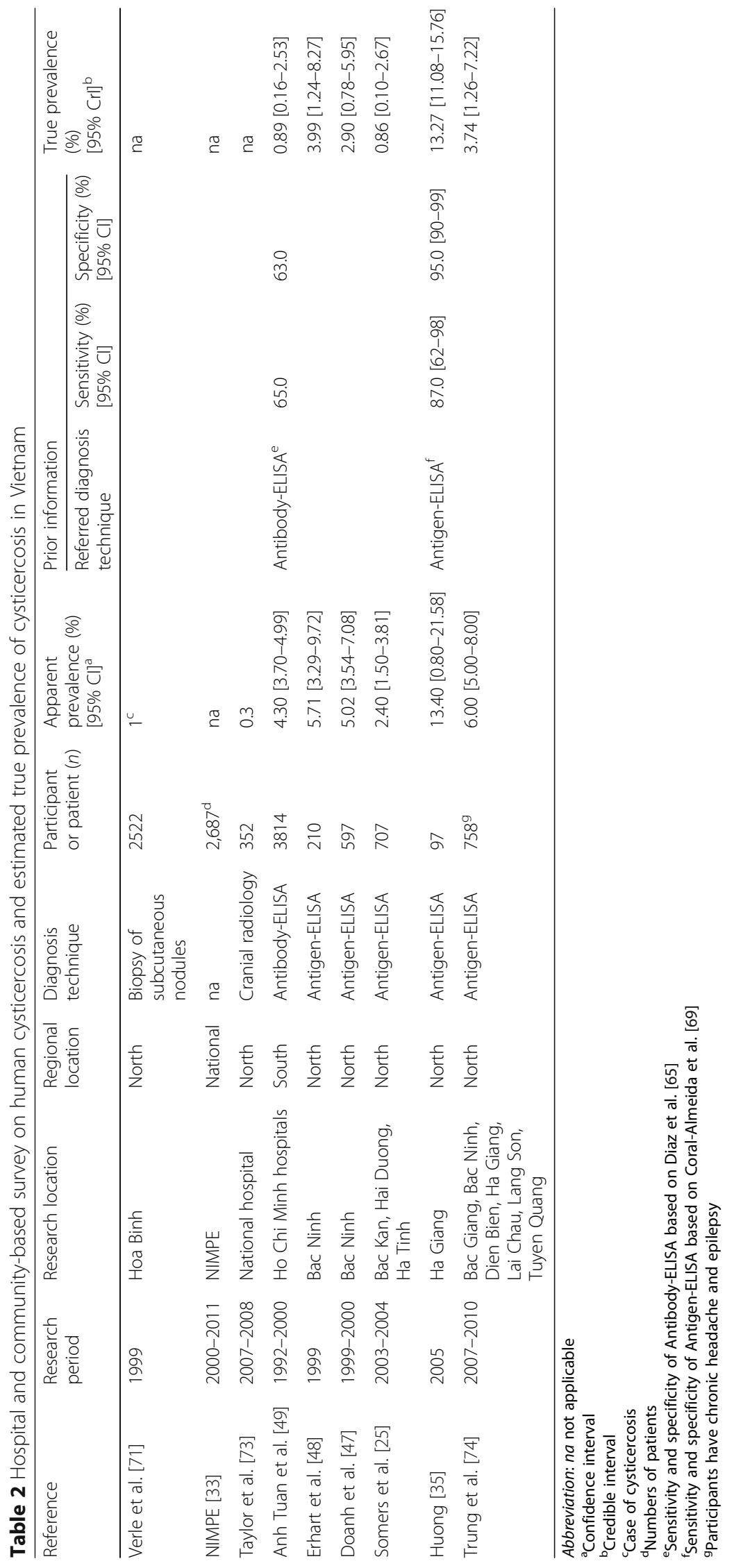




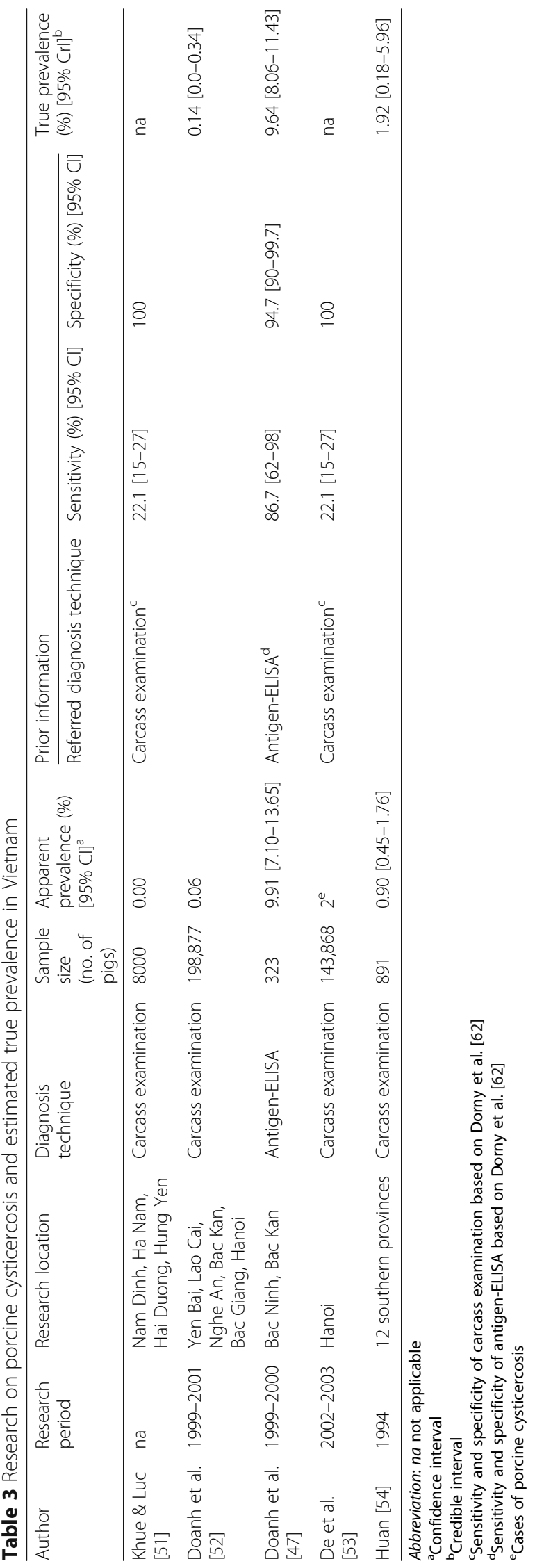



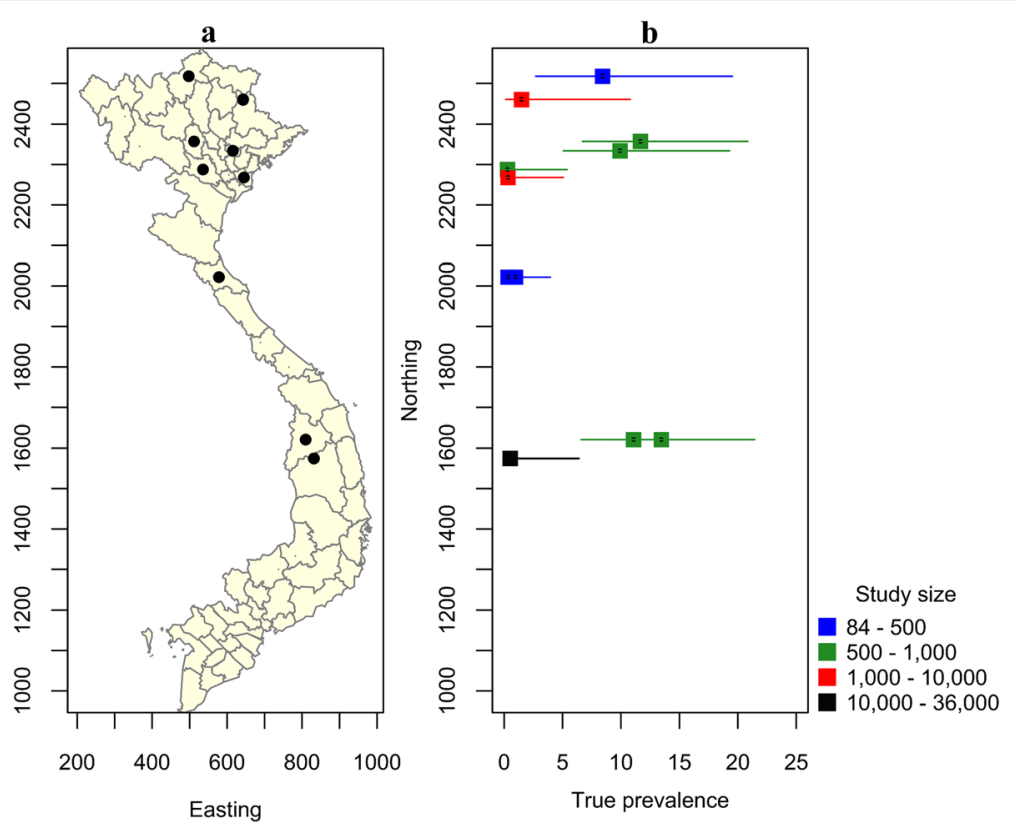

Fig. 2 Studies of the prevalence of taeniasis in Vietnam, 1999 to present. a Map of Vietnam showing the location of studies described in the text. b Error bar plot showing the known true prevalence of taeniasis (and their $95 \%$ confidence intervals) as a function of the northing coordinate of the province in which the study was carried out

account for a higher proportion of taeniasis cases compared with females. In addition, adults of working age have been shown to have a higher risk of infection compared with other age groups [34, 38-42].

Before the first report of $T$. asiatica in the North of Vietnam in 2001 [43] the relative proportions of $T$. saginata to T. solium cases were 80 and $22 \%$, respectively, based on morphological identification of purged proglottids in stools [44]. Later, Doanh et al. [32] confirmed the presence of $T$. asiatica in six samples from Bac Ninh province using molecular diagnostic techniques. Somers et al. [45] showed that T. asiatica dominated taeniasis cases (55\%) in the northern provinces of Vietnam, followed by T. saginata (38.5\%) and T. solium (6.2\%). Between 2005 and 2006 Taenia proglottids from 65 individuals were identified from 19 provinces in the North. This study confirmed the presence of $T$. asiatica and T. saginata but not $T$. solium [42]. Huong [35] confirmed the presence of $T$. asiatica in three out of five samples collected in $\mathrm{Ha}$ Giang province. De \& Hoa [46] identified T. saginata proglottids in eight patients in the Central provinces, the Central Highlands and the South of Vietnam using molecular diagnostic techniques. No T. solium or T. asiatica proglottids were identified. The latter study is the only report describing the species of human tapeworms occurring in Central and South Vietnam. A limited number of studies indicate that $T$. asiatica is restricted to the north of the country.

\section{Human cysticercosis}

A review of the literature indicates that human cysticercosis has been reported in 55 of the 63 provinces of Vietnam. Data on the AP and estimated TP of T. solium cysticercosis in humans is summarized in Table 2. The prevalence of infection varies widely between study areas ranging from no cases found in the central province of Hai Duong to $13 \%$ (95\% CI: $0.80-22 \%$ ) in Ha Giang province in the north (Table 2).

In the 6-year period from 2006 to 2011, an estimated 250 to 400 patients from 34 provinces in North Vietnam were hospitalized and treated for $T$. solium cysticercosis by the NIMPE annually. The majority of these patients were from Hai Phong, Thanh Hoa, Ha Noi, Bac Giang and Bac Ninh provinces [33]. Between 1999 and 2000, the results of three village-based surveys conducted in Bac Ninh province showed an AP of cysticercosis of 5.0\% (range from 2.2 to $7.2 \%$ ) [47]. A cross-sectional study carried out in the same province by Erhart et al. [48] in 1999 showed that $12(5.7 \%)$ out of 210 individuals in Ty Dien village had circulating T. solium cysticercosis antigen and nine were confirmed as having NCC using computed tomography scanning. A short report on the situation of taeniasis and cysticercosis in Vietnam by De stated that of 4017 people diagnosed with helminth infection by NIMPE, 633 were also positive for cysticercosis. No further details of area-specific prevalence or methods of diagnosis were provided in this report. 
In the South of Vietnam, a sero-epidemiological survey carried out in hospitals located in Ho Chi Minh City from 1992 to 2000 involving 3814 people of all ages mainly originating from South Vietnam found that 4.3\% were positive for cysticercosis. Patients that were residents of Ho Chi Minh City and the province of An Giang accounted for the highest proportions of study subjects that were cysticercus positive, i.e. 20 and 14\%, respectively [49]. The study of Anh Tuan et al. [49] is the only study documenting the prevalence of $T$. solium cysticercosis in the South of Vietnam, and to the best of our knowledge, there are no reports of the prevalence of T. solium cysticercosis for Central Vietnam (Fig. 3). A single case report describes $T$. solium NCC in three ethnic minority patients from Quang Tri and Quang Ngai province hospitalized at Hue Central Hospital in 2012. Each had seizures and headaches [50].

NIMPE [33] reported that among 2687 T. solium cysticercosis cases, the proportion of males was significantly greater than that of the proportion females. In addition, study subjects that were between 30 and 60 years of age were over-represented. Anh Tuan et al. [49] reported that in Ho Chi Minh City, males comprised 56\% and adults comprised $86 \%$ of the 163 study subjects that were positive to an Ab-ELISA for T. solium cysticercosis. The consumption of raw vegetables, drinking unboiled water, not washing hands before eating and outdoor defecation were found to be risk factors for $T$. solium cysticercosis in this study. Undoubtedly, utilization of night-soil for fertilizing crops is a major contributing factor to $T$. solium cysticercosis and this practice has been reported in both the North and South of Vietnam.

\section{Porcine cysticercosis}

Data on the prevalence of porcine cysticercosis at the national level is lacking, fragmented and/or out of date. Surveys on porcine cysticercosis were carried out primarily in the north and mostly in Hanoi slaughterhouses (Fig. 4). Data on the AP and estimated TP of porcine cysticercosis is summarized in Table 3. There were no cases of cysticercosis among approximately 8000 carcasses examined at slaughterhouses in Nam Dinh and Ha Nam, Hai Duong and Hung Yen provinces [51] (Table 3). Between 1999 and 2001, 0.06\% of 198,887 pig carcasses examined in five provinces in the North and one province in Central Vietnam were identified as infected [52]. In a community-based survey carried out between 1999 and 2000 in five villages in Bac Kan and Bac Ninh province (known to be highly endemic foci for $T$. solium cysticercosis) an apparent cysticercosis seroprevalence of 9.91\% (95\% CI: 7.10-14\%) was reported in pigs [47] using Ag-ELISA. In a survey from 2002 to 2003 on porcine cysticercosis was carried out in abattoirs and markets in Hanoi, in which only two out of 143,868 carcasses examined were found to be infected with T. solium [53].

In South Vietnam, there is a paucity of information on the prevalence of cysticercosis in pigs (Fig. 4). A single
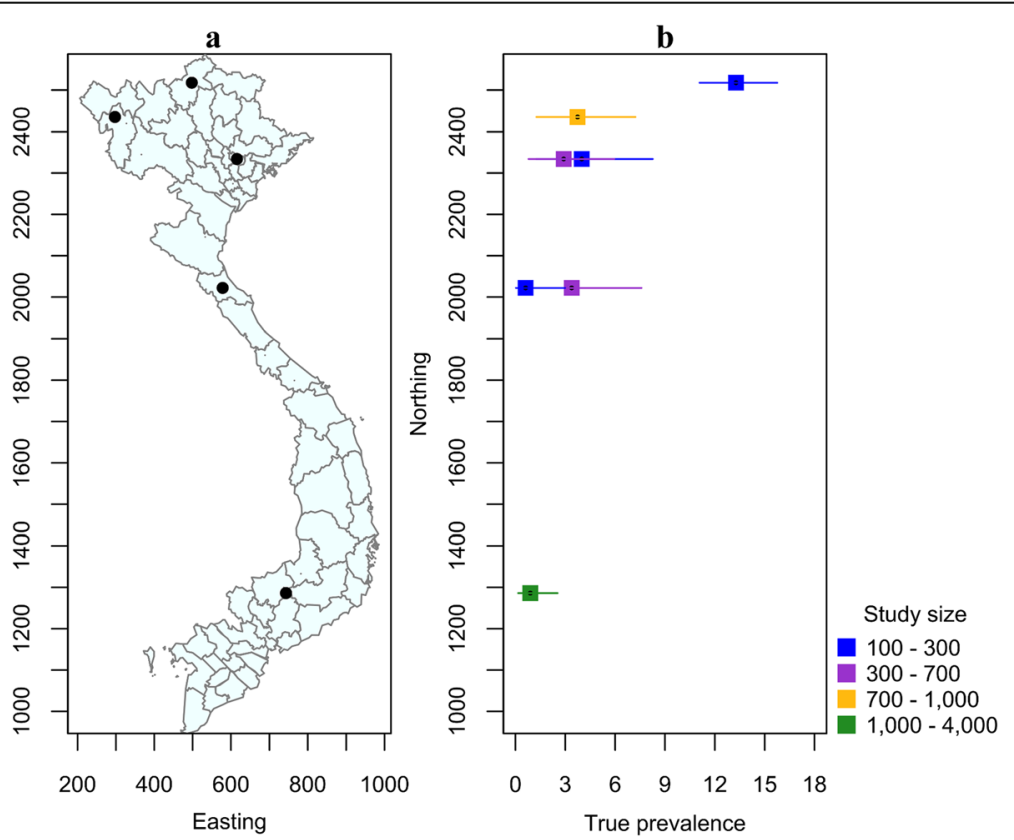

Fig. 3 Studies of the prevalence of human T. solium cysticercosis in Vietnam, 1992 to present. a Map of Vietnam showing the location of studies described in the text. b Error bar plot showing the known true prevalence of T. solium cysticercosis in humans (and their 95\% confidence intervals) as a function of the northing coordinate of the province in which the study was carried out 

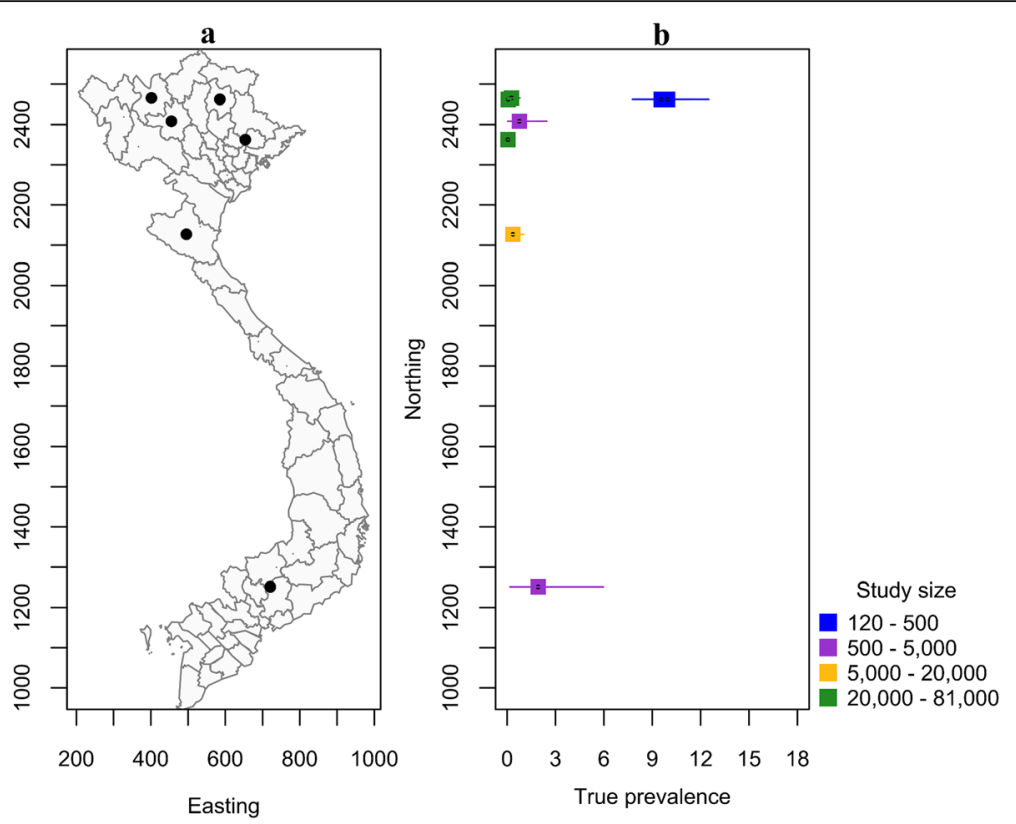

Fig. 4 Studies of the prevalence of T. solium cysticercosis in pigs in Vietnam, 1994 to present. a Map of Vietnam showing the location of studies described in the text. $\mathbf{b}$ Error bar plot showing the known true prevalence of T. solium cysticercosis pigs (and their $95 \%$ confidence intervals) as a function of the northing coordinate of the province in which the study was carried out

study conducted in 1994 showed that $0.90 \%$ of 891 pigs from 18 districts located in 12 southern provinces were T. solium cysticercosis positive [54].

\section{Trichinellosis}

In Vietnam, the first human case of trichinellosis was identified in 1968 in a group of people consuming pork from Lao PDR [55]. Since then, there have been five reported outbreaks in four provinces (Table 4 and Fig. 5). In 1970, an outbreak in Mu Cang Trai District, Yen Bai province (in the north) resulted in 30 cases of trichinellosis, including four deaths [56]. In 2002, 22 people in Tuan Giao District, Dien Bien province were confirmed infected of which two people died. In 2008, in Bac Yen District, Son La province an outbreak of trichinellosis arising from consumption of undercooked pork from domestic pigs resulted in 23 cases of trichinellosis and two deaths [57]. Similarly, 24 out of 27 individuals acquired trichinellosis after eating raw pork in a mountainous region of Thanh Hoa province in 2012. Of these, six went on to develop serious symptoms [58]. Following the outbreak of human trichinellosis in Son La province in 2008, $20 \%$ of free-roaming pigs were reported seropositive for Trichinella antibodies in the four villages in which the human outbreaks occurred [59]. Of the 206 muscle samples that were sero-positive, Trichinella larvae were recovered from 11 samples and identified as $T$. spiralis using multiplex PCR. The proportion of Trichinella seropositive wild boars and rats in Son La and Dien Bien province was 3.2\% (2 positive out of 62 tested) and $2.8 \%$ (23 positive out of 820 tested), respectively, and $4 \%$ (5 positive out of 125 tested) of dogs were also found to be seropositive to Trichinella [60]. Surprisingly, none of the 261 confined wild boars resident on seven farms or 98 cats in these two provinces were positive [59]. In the same study, T. spiralis larval burdens

Table 4 Trichinellosis outbreaks in Vietnam since 1970

\begin{tabular}{|c|c|c|c|c|c|}
\hline Outbreak (year) & Location (province) & Geographical location & Trichinella spp. & Infected toll (no. of people) & Death toll (no. of people) \\
\hline 1970 & Yen Bai & Northwest & na & 26 & 4 \\
\hline 2001 & Dien Bien & Northwest & na & 22 & 2 \\
\hline 2004 & Dien Bien & Northwest & na & 20 & na \\
\hline 2008 & Son La & Northwest & T. spiralis (animals) & 22 & 2 \\
\hline 2012 & Thanh Hoa & North Central Coast & T. spiralis (humans) & 24 & na \\
\hline
\end{tabular}

Adjusted from: De et al. [58]; Vu Thi et al. [59, 61]; Taylor et al. [75] 


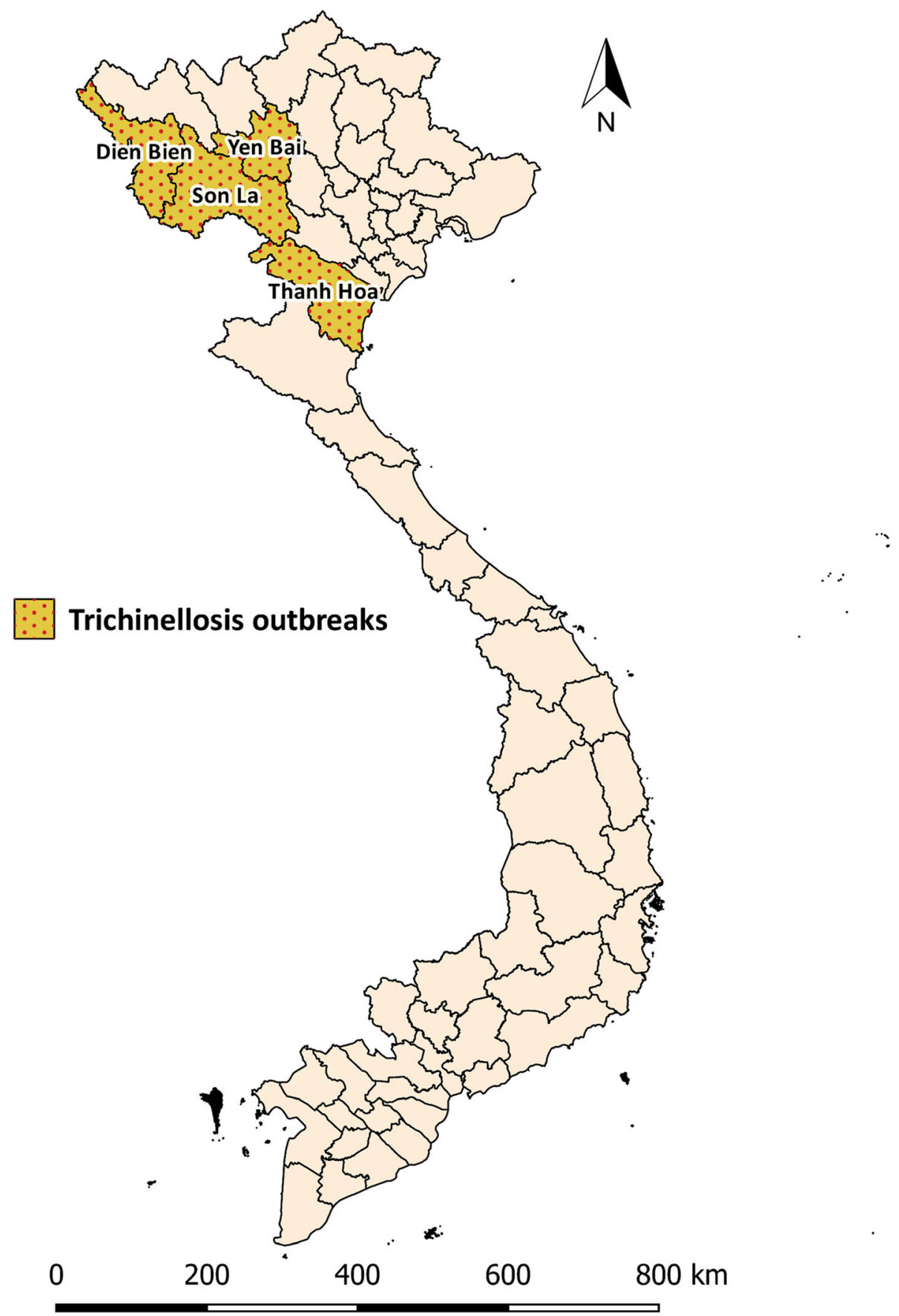

Fig. 5 Map of trichinellosis outbreaks in Vietnam. Choropleth map showing provinces in which trichinellosis outbreaks have occurred since 1970

quantified using multiplex PCR were relatively low at 0.1 to 0.3 larvae/gram of muscle in wild boars and 0.6 larvae/gram of muscle in rats [59]. Until now, the only species of Trichinella isolated in domestic and sylvatic hosts in North Vietnam has been T. spiralis [61]. The habit of eating traditional dishes prepared using under-cooked and/or raw game meat (wild pork) at special events such as the lunar New Year, weddings and funeral events is the reason attributed to Trichinella outbreaks in North Vietnam. Leaving pigs to free roam, and feeding raw and/or left-over food to pigs has been blamed for the transmission of Trichinella in the region where medical personnel often lack knowledge about trichinellosis and its clinical symptoms. Moreover, in this area of Vietnam healthcare services are difficult to access [60]. There are no reports available on the seroprevalence of Trichinella 
infection in wild boars and domestic swine from other regions in Vietnam.

\section{Discussion}

Our study has shown that taeniasis and T. solium cysticercosis occurs in 60 of the 63 provinces of Vietnam (Fig. 6). While data on the prevalence of taeniasis, cysticercosis and trichinellosis are available for North Vietnam, the relatively small number of studies carried out in the center and south of Vietnam mean that it is difficult to draw definitive conclusions about the prevalence of these conditions in these areas of the country.

Across all of the cited studies utilizing microscopy to detect taeniasis, TP estimates were greater than the AP

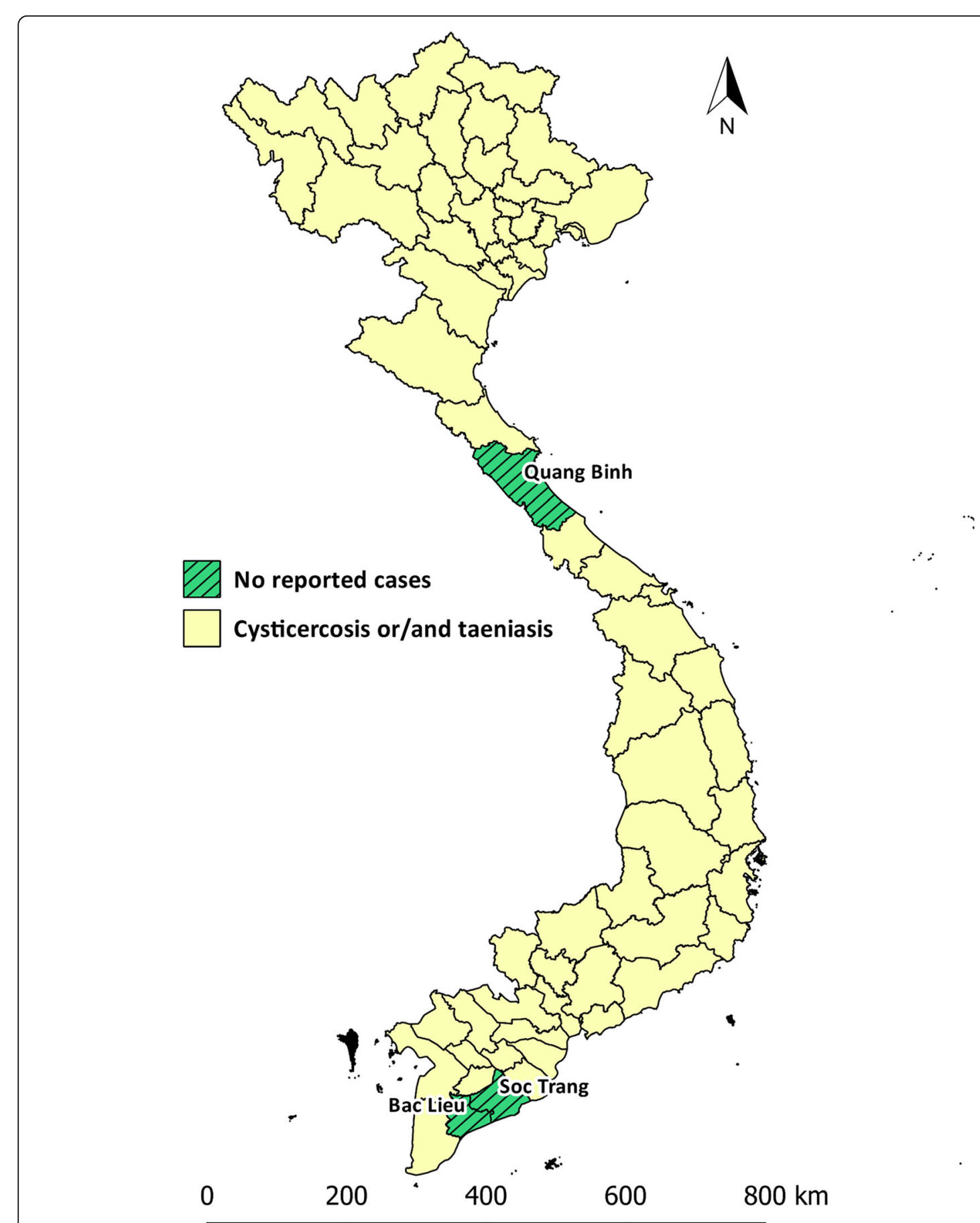

Fig. 6 Distribution of human T. solium cysticercosis and taeniasis. Choropleth map of Vietnam showing provinces in which cysticercosis and/or taeniasis have been identified in humans at present 
estimates (Table 1). For example, the TP of taeniasis in Ha Giang [35] at $8.00 \%$ (95\% CrI: $2.70-20 \%)$ was greater than that of the AP $(6.00 \%, 95 \%$ CI: $2.60-13 \%)$. Similarly the estimated TP in Chuong [37] was 0.52\% (95\% CrI: $0.03-6.0 \%$ ) nearly twice that of the AP (Table 1 ). It is highly likely that the prevalence of taeniasis has been grossly underestimated due to the utilization of microscopy-based examinations for the presence of Taenia spp. eggs using Kato-Katz and/or Formalin-Ether Concentration techniques. The difference between the reported apparent and estimated TP is likely to be a result of the low Se of the microscopy-based methods to detect light taeniasis infections [62] and intermittent proglottid shedding [63]. It is also noteworthy that microscopic-based techniques are incapable of specieslevel identification of Taenia spp. in stool as the eggs are morphologically identical.

In contrast to the TP of taeniasis, the TP estimates for human cysticercosis were lower than the AP estimates in across all of the cited studies (Table 2). The TP of cysticercosis in the study by Anh Tuan et al. (2001) [49] in South Vietnam was 0.89\% (95\% CrI: $0.16-2.53 \%)$, much lower than the AP of $4.30 \%$ (95\% CI: $3.70-4.99 \%)$. It should be noted that Ab-ELISA with a relatively poor $\mathrm{Sp}$ were utilized for this study; thus, overestimation of AP on human cysticercosis in this region may occur since Ab-ELISAs cross-react with Echinococcus granulosus (hydatid disease), Schistosoma spp. Angiostrongylus cantonensis, Spirometra spp., Fasciola spp. and Hymenolepis spp. [64, 65]. On the other hand, Ab-ELISAs provide a measure of $T$. solium cysticercosis exposure, not T. solium cysticercosis infection. As a result, Ab-ELISAs may also detect cases of transitional antibody exposure to $T$. solium $[66,67]$ and/or immunologically persistent antibody [68], hence it may overestimate both the AP and TP of active T. solium cysticercosis in a community.

Ag-ELISA is known to have an acceptable Se of 87\% (95\% CI: $62-98 \%$ ) and Sp of 95\% (95\% CI: $90-$ 99\%) for the detection of T. solium cysticercosis active infections in humans [69]; however, the estimated TP of human cysticercosis was lower than that of the AP in all of the cited studies in this review. This can be attributed to the uncertainty of the $\mathrm{TP}$ of human cysticercosis across study locations couple with imperfect of the assay (95\%). Ag-ELISAs are superior to Ab-ELSIAs in term of detecting active infection of $T$. solium cysticercosis; however, since both assays are genus-specific, this does not necessarily apply to pigs. In pigs, the assays are known to cross-react with antigens of Taenia hydatigena, a common tapeworm of swine in Vietnam [70]. Therefore, the AP of T. solium cysticercosis in village pigs in Bac Kan and Bac Ninh province of 9.9\% (95\% CI: 6.1-15\%) is likely to be an overestimate. Of 29 pigs positive to Ag-ELISA, only five pigs were found to be infected with cysts of $T$. solium and ten pigs with cysts of $T$. hydatigena [52].

From the data presented in this systematic review, it appears that abattoir-based surveys may be biased and are likely to result in an underestimation of the risk of taeniasis and cysticercosis in humans because the majority pigs presented to these abattoirs were sourced from commercial piggeries. On the other hand, village-reared pigs are likely to be slaughtered locally and therefore represent a greater risk of infection for humans. Moreover, post-mortem inspection-based techniques have a low reported Se of $22 \%$ for the detection of cysticerci in meat [62] and are likely to lead to a further underestimate of the TP of T. solium cysticercosis.

The prevalence of taeniasis and porcine cysticercosis in Vietnam are most likely to be underestimated whereas the prevalence of human cysticercosis is likely to be overestimated. In relative terms, the prevalence of porcine cysticercosis was low compared with taeniasis. Since microscopic-based diagnosis is genus-specific, it is likely that $T$. saginata and potentially $T$. asiatica accounted for a proportion of Taenia infections in humans. Thus, further epidemiological surveys on bovine cysticercosis, and village-based surveys on porcine cysticercosis is necessary to fully unveil the entire epidemiological picture of taeniasis in humans. Gathered risk factors for porkborne zoonoses includes the consumption of raw/undercooked pork, beef and vegetables and the utilization of night-soil for fertilization of local produce.

\section{Conclusion}

Although there are detailed data available on the prevalence of food-borne parasitic zoonoses relating to taeniasis, cysticercosis and trichinellosis in the north of Vietnam, by contrast, little to no data are available for the central and southern areas of the country. The utilization of copro-diagnostic tests for human taeniasis and post-mortem diagnosis of porcine $T$. solium cysticercosis likely resulted in an underestimation of the TP of infection in these hosts. On the other hand, genus-specific immunodiagnostic tests with imperfect Sp likely resulted in an overestimation of human and porcine cysticercosis. Moreover, sampling methods that were conducted without a clearly defined randomized design limits our ability to make accurate estimates of the TP of these infections in either humans or pigs in each of the three regions of Vietnam. In addition, studies based on slaughterhouse surveillance are believed not to reflect the true risk posed to humans, given the majority of the population live in rural, remote communities. Future 
surveillance aimed at conducting random crosssectional village-based surveys of taeniasis and $T$. solium cysticercosis in humans and cysticercosis in pigs and cattle using improved molecular and immunodiagnostic methods, will shed further light on the epidemiology of these food-borne zoonoses among rural communities. This information will assist local government and residents to develop appropriate risk mitigation efforts to reduce the burden of these infections for the betterment of market access and public health.

\section{Additional file}

Additional file 1: PRISMA 2009 checklist. (DOC 63 kb)

\section{Abbreviations}

AP: Apparent prevalence; GDP: Gross domestic product:

NNC: Neurocysticercosis; PRISMA: Preferred reporting items for systematic reviews and meta-analyses; Se: Sensitivity; Sp: Specificity; TP: True prevalence

\section{Acknowledgements}

We sincerely thank Dr Nguyen Van Tuan from Institute of Malariology Parasitology and Entomology Quy Nhon (IMPE-QN) for providing materials for this review. We are grateful to Tay Nguyen University, Vietnam for in-kind support.

\section{Funding}

This research was self-funded.

\section{Availability of data and materials}

The data supporting the conclusions of this article are included within the article.

\section{Authors' contributions}

DNN designed study, analyzed data and wrote manuscript; MAS: designed study, assisted with analyses of data and edited paper; RJT designed, supervised study and edited paper. All authors read and approved the final manuscript.

\section{Competing interests}

The authors declare that they have no competing interests.

\section{Consent for publication}

Not applicable.

\section{Ethics approval and consent to participate}

This study was reviewed and approved by the Behavioural and Social Sciences/ Human Ethics Sub-committee, the University of Melbourne (reference number 1443512).

\section{Publisher's Note}

Springer Nature remains neutral with regard to jurisdictional claims in published maps and institutional affiliations.

\section{Received: 25 November 2016 Accepted: 10 March 2017}

Published online: 21 March 2017

\section{References}

1. FAO/WHO [Food and Agriculture Organization of the United Nations/World Health Organization]. Multicriterial-based ranking for risk management of food-borne parasites. Microbiologycal Risk Assessment Series No 23. WHO press; 2014

2. Torgerson PR, Devleesschauwer B, Praet N, Speybroeck N, Willingham AL, Kasuga F, et al. World Health Organization Estimates of the Global and
Regional Disease Burden of 11 Foodborne Parasitic Diseases, 2010: A Data Synthesis. PLoS Med. 2015;12:1-22.

3. Murrell KD, Dorny P, Flisser A, Geerts S, Kyvsgaard NC, McManus DP, et al. WHO/ FAO/OIE Guidelines for the surveillance, prevention and control of taeniosis/ cysticercosis. OIE (World Organisation for Animal Health), WHO (World Health Organization) and FAO (Food and Agriculture Organization); 2005.

4. Schantz PM, Cruz M, Sarti E, Pawlowski ZS. Potential eradicability of taeniasis and cysticercosis. Bull PAHO. 1993;27:397-403.

5. World Health Organization. Atlas: epilepsy care in the world. Geneva: WHO express; 2005

6. Pozio E. World distribution of Trichinella spp. infections in animals and humans. Vet Parasitol. 2007;149:3-21.

7. Bowles J, McManus DP. Genetic characterization of the Asian Taenia, a newly described taeniid cestode of humans. Am J Trop Med Hyg. 1994;50: 33-44.

8. De NV, Le TH, Lien PTH, Eom KS. Current status of taeniasis and cysticercosis in Vietnam. Korean J Parasitol. 2014:52:125-9.

9. Ito A, Nakao M, Wandra T. Human taeniasis and cysticercosis in Asia. Lancet. 2003:362:1918-20.

10. Eom KS, Jeon HK, Rim HJ. Geographical distribution of Taenia asiatica and related species. Korean J Parasitol. 2009:47:S115-24.

11. Gonzales I, Rivera JT, Garcia HH. Cysticercosis Working Group in Peru. Pathogenesis of Taenia solium taeniasis and cysticercosis. Parasite Immunol. 2016:38:136-46.

12. Carabin $H$, Ndimubanzi PC, Budke CM, Nguyen H, Qian Y, Cowan LD, et al. Clinical manifestations associated with neurocysticercosis: a systematic review. PLoS Negl Trop Dis. 2011;5:e1152.

13. White AC. Neurocysticercosis: updates on epidemiology, pathogenesis, diagnosis, and management. Annu Rev Med. 2000;51:187-206.

14. Pozio E, Hoberg E, La Rosa G, Zarlenga DS. Molecular taxonomy, phylogeny and biogeography of nematodes belonging to the Trichinella genus. Infect Genet Evol. 2009:9:606-16.

15. Pozio E. Taxonomy of Trichinella and the epidemiology of infection in the Southeast Asia and Australian regions. Southeast Asian J Trop Med Public Health. 2001;32:129-32.

16. Takahashi Y, Mingyuan L, Waikagul J. Epidemiology of trichinellosis in Asia and the Pacific Rim. Vet Parasitol. 2000;93:227-39.

17. Robertson L, Sprong H, Ortega Y, van der Giessen JWB, Fayer R. Response to Galán-Puchades and Fuentes: Taenia asiatica: neglected-but not forgotten-and almost certainly being quietly globalised. Trends Parasitol. 2014;30:56-7.

18. Wu HW, Ito A, Ai L, Zhou XN, Acosta LP, Lee WA. Cysticercosis/taeniasis endemicity in Southeast Asia: Current status and control measures. Acta Trop Elsevier BV. 2015;165:121-32.

19. Hoang Nam. Vietnam image of the community of 54 Ethic Groups. Aff Comm Ethn Minor. 2016 [cited 2016 Sep 16]. Available from: http://english. ubdt.gov.vn/vietnam-image-of-the-commtnity-of-54-ethnic-groups.htm.

20. General Statistics Office of Vietnam. Statistical Data. Gen Stat Off Vietnam. 2015 [cited 2015 Dec 22]. Available from: http://www.gso.gov.vn/

21. United Nations. Human Development Index and its components. United Nations Dev Prog. 2014 [cited 2015 Dec 23]. Available from: http://hdr.undp. org/en/composite/HDI.

22. World Bank. World Development Indicators. World Bank. 2014 [cited 2015 Dec 23]. Available from: http://databank.worldbank.org/data/reports. aspx? source=world-development-indicators.

23. Carrique-Mas JJ, Bryant JE. A review of foodborne bacterial and parasitic zoonoses in Vietnam. Ecohealth. 2013 [cited 2016 Sep 14].

24. Uga S, Hoa NT, Noda S, Moji K, Cong L, Aoki Y, et al. Parasite egg contamination of vegetables from a suburban market in Hanoi, Vietnam. Nepal Med Coll J. 2009;11:75-8

25. Somers R, Dorny P, Nguyen VK, Dang TCT, Goddeeris B, Craig PS, et al. Taenia solium taeniasis and cysticercosis in three communities in north Vietnam. Trop Med Int Heal. 2006;11:65-72.

26. Moher D, Liberati A, Tetzlaff J, Altman DG, Oxman AD, Cook DJ, et al. Preferred reporting items for systematic reviews and meta-analyses: The PRISMA Statement. J Clin Epidemiol Elsevier. 2009:62:1006-12.

27. Rogan W, Gladen B. Estimating prevalence from results of a screening test. Am J Epidemiol. 1978;107:71-6.

28. Messam L, Branscum A, Collins M, Gardner I. Frequentist and Bayesian approaches to prevalence estimation using examples from Johne's disease. Anim Heal Res Rev. 2008;9:1-23. 
29. Branscum AJ, Gardner IA, Johnson WO. Bayesian modeling of animal- and herd-level prevalences. Prev Vet Med. 2004;66:101-12.

30. Lunn DJ, Thomas A, Best N, Spiegelhalter D. WinBUGS - a Bayesian modelling framework: Concepts, structure, and extensibility. Stat Comput. 2000;10:325-37

31. Wakefield JC, Best NG, Waller L. Bayesian approaches to disease mapping. In: Elliott P, Wakefield J, Best N, Briggs D. Spatial Epidemiology: Methods and Applications. London: Oxford University Press; 2000. pp. 118.

32. Doanh NQ, Holland W, Tam PT, De NV, Hoa LT, Hoan DH. The results of the studies on situation of humans and pigs contracting disease that cestode worm and its larvae cause for. Sci Technol J Agric Rural Dev. 2006;4/2006: 56-68.

33. NIMPE. Taeniasis and Cysticercosis. Rev. Work. helminthiasis Control Act. period 2006-2011 Implement. Work. period 2012-2015. Ministry of Health of Vietnam; 2012

34. Vien HV, Hung NM, Thach DTC, Thuan LK, Dung DT, Hop NT, et al. Investigate Taenia infection in two communes of Tan Son District, Phu Tho Province. J Malar Parasite Dis Control. 2012;1-2012:69-74.

35. Huong NT. Taeniasis and cysticercosis in a selected group of inhabitants from a mountainous province in North Vietnam. Belgium: Prince Leopold Institute of Tropical Medicine, Antwerpen (Antwerp); 2006.

36. Phuong VTB, Tra HTU, Duyen NT. Research on intestinal parasitic infection on patients in parasitology department in the hospital of thai binh medical university hospital from 2008-2010. Y Hoc Thanh Pho Ho Chi Minh. 2012;16:7-10.

37. Van Chuong N. Status of helminthic infection in humans in 14 provinces spanning the Central and Central Highland regions of Vietnam. IMPE-QN. 2005 [cited 2016 Mar 1]. Available from: http://www.impe-qn.org.vn/impeqn/vn/portal/InfoDetail.jsp?area=58\&cat=1068\&ID=597.

38. Van Tuan B, Chuong N Van. Situation of Taenia saginata infestation and effectiveness of some control measures in Dak Mon commune (Dak Glei) and Yaxier commune (Sa Thay), Kon Tum provinice. J Malar Parasite Dis Control. 2014;1-2014:41-8.

39. Van Chuong N, Van Tuan B, Van Kha N, Khanh NH. Research on Taenia saginata infection situation in some areas of Kon Tum province. J Paractical Med. 2011;796:156-9.

40. Sieu TPM, Ly LTC. Study of some characteristics of taeniasis (Taenia saginata) In Ho Chi Minh City. Y Hoc Thanh Pho Ho Chi Minh. 2013;17:126-9.

41. Ho ST. Study on genotype of pathogen, clinical, subclinical symptoms, treatment efficacy for taeniasis and cysticercosis patients in National Institute of Malariology, Parasitology and Entomology 2007-2010. Hanoi: Hanoi Medical University; 2013.

42. Vien HV, Le DD, Manh N, Tan HV, Nguyen DH, Nhung VT. Identication of tapeworm Taenia spp. and cysticercosis in human by Multiplex-PCR. J Malar Parasite Dis Control. 2008;1:62-8.

43. De NV, Hoa LT, Doanh NQ, Ngoc NB, Cong LD. Report on a new species of Taenia (Taenia asiatica) in Hanoi, Vietnam. J Malar Parasite Dis Control. 2001; 3:80-5.

44. Willingham AL, De NV, Doanh NQ, Cong D, Dung TV, Dorny $P$, et al. Current status of cysticercosis in Vietnam. Southeast Asian J Trop Med Public Health. 2003;34 Suppl 1:35-50

45. Somers R, Dorny P, Geysen D, Nguyen LA, Thach DC, Vercruysse J, et al. Human tapeworms in north Vietnam. Trans R Soc Trop Med Hyg. 2007;101: 275-7.

46. De NV, Hoa LT. Molecular identification of human Taenia saginata and Taennia asiatica in Vietnam. Vietnam J Med. 2006;1:42-50.

47. Doanh NQ, Kim NT, De NV, Lung NN. Result of survey on taeniasis and cysticercosis humans and pigs in Bac Ninh and Bac Kan provinces. Vet Sci Tech. 2002;9:46-9.

48. Erhart A, Dorny P, De NV, Vien HV, Thach DC, Toan ND, et al. Taenia solium cysticercosis in a village in northern Viet Nam: Sero-prevalence study using an ELISA for detecting circulating antigen. Trans R Soc Trop Med Hyg. 2002; 96:270-2.

49. Anh Tuan P, Dung TTK, Nhi VA. Sero-epidemiological investigation of cysticercosis in the southern provinces. J Malar Parasite Dis Control. 2001:4:81-7.

50. Trung PT, Doan BV. Neurocysticercosis: Report of three cases in infectious disease department of Hue Central Hospital. Y Hoc Thanh Pho Ho Chi Minh. 2013;17:110-4.

51. Khue PV, Luc P. Veterinary Parasitology. Hanoi: Hanoi Agricultural Publishing House; 1996.
52. Doanh NQ, Holland W, Vecruyce J, De NV. Results of survey on cysticercosis pig in some nothern provinces in Vietnam. J Malar Parasite Dis Control. 2002;6:76-82.

53. De NV, Chau LV, Son DT, Chuyen LT, Hop NT, Vien HV, et al. Taenia solium survey in Hanoi. J Malar Parasite Dis Control. 2004;6:93-9.

54. Huan LV. Parasitic helminths in pigs in several southern provinces and preventative measures. Ph.D. Hanoi: National Institution of Veterinary Research; 1994.

55. Phan L. Parasite and veterinary parasitic diseases. Hanoi: Hanoi Agriculture Publishing House; 1997.

56. De NV, Nga VT, Dorny P, Trung NV, Minh PN, Dung DT, et al. Trichinellosis in Vietnam. Am Soc Trop Med Hyg. 2015;92:1265-70.

57. Van Nguyen C, Vu Thi N, Nguyen TC. Result of survey and solution for trichinellosis in pigs in Bac Ha District, Son La Province. Vet Sci Tech. 2012; 19:50-6.

58. De NV, Trung NV, Ha NH, Nga VT, Ha NM, Thuy PT, et al. An outbreak of trichinosis with molecular identification of Trichinella sp. in Vietnam. Korean J Parasitol. 2012;50:339-43.

59. Vu Thi N, Nguyen VD, Praet N, Claes L, Gabriël S, Huyen NT, et al. Trichinella infection in wild boars and synanthropic rats in northwest Vietnam. Vet Parasitol. 2014;200:207-11.

60. Vu Thi N, De NV, Praet N, Claes L, Gabriël S, Dorny P. Seroprevalence of trichinellosis in domestic animals in northwestern Vietnam. Vet Parasitol. 2013;193:200-5.

61. Vu Thi N, Trung DD, Litzroth A, Praet N, Nguyen Thu H, Nguyen Thu H, et al. The hidden burden of trichinellosis in Vietnam: a postoutbreak epidemiological study. Biomed Res Int. 2013;2013:149890.

62. Dorny P, Phiri IK, Vercruysse J, Gabriel S, Willingham IAL, Brandt J, et al. A Bayesian approach for estimating values for prevalence and diagnostic test characteristics of porcine cysticercosis. Int J Parasitol. 2004;34:569-76.

63. Smyth JD, McManus DP. The physiology and biochemistry of cestodes. Cambridge: Cambridge University Press; 2007.

64. Rodriguez S, Wilkins P, Dorny P. Immunological and molecular diagnosis of cysticercosis. Pathog Glob Health. 2012;106:286-98.

65. Diaz JF, Verastegui M, Gilman RH, Tsang VC, Pilcher JB, Gallo C, et al. Immunodiagnosis of human cysticercosis (Taenia solium): a field comparison of an antibody-enzyme-linked immunosorbent assay (ELISA), an antigen-ELISA, and an enzyme-linked immunoelectrotransfer blot (EITB) assay in Peru. The cysticercosis working group in. Am J Trop Med Hyg. 1992;46:610-5.

66. Deckers N, Dorny P. Immunodiagnosis of Taenia solium taeniosis/ cysticercosis. Trends Parasitol. 2010;26:137-44.

67. Garcia HH, Gonzalez AE, Gilman RH, Palacios LG, Jimenez I, Rodriguez S, et al. Short report: transient antibody response in Taenia solium infection in field conditions-a major contributor to high seroprevalence. Am J Trop Med Hyg. 2001;65:31-2.

68. Garcia HH, Gilman RH, Catacora M, Verastegui M, Gonzalez AE, Tsang VCW. Serologic evolution of neurocysticercosis patients after antiparasitic therapy. J Infect Dis. 1997:175:486-9.

69. Coral-Almeida M, Gabriël S, Abatih EN, Praet N, Benitez W, Dorny P. Taenia solium human cysticercosis: A systematic review of sero-epidemiological data from endemic zones around the world. PLoS Negl Trop Dis. 2015;9:1-20.

70. Dorny P, Brandt J, Zoli A, Geerts S. Immunodiagnostic tools for human and porcine cysticercosis. Acta Trop. 2003;87:79-86.

71. Verle P, Kongs A, De NV, Thieu NQ, Depraetere K, Kim HT, et al. Prevalence of intestinal parasitic infections in northern Vietnam. Trop Med Int Heal. 2003;8:961-4.

72. Praet N, Verweij JJ, Mwape KE, Phiri IK, Muma JB, Zulu G, et al. Bayesian modelling to estimate the test characteristics of coprology, coproantigen ELISA and a novel real-time PCR for the diagnosis of taeniasis. Trop Med Int Heal. 2013;18:608-14.

73. Taylor WR, Nguyen $\mathrm{K}$, Nguyen D, Nguyen $\mathrm{H}$, Horby P, Nguyen $\mathrm{HL}$, et al. The spectrum of central nervous system infections in an adult referral hospital in Hanoi, Vietnam. PLoS One. 2012;7:e42099.

74. Trung DD, Praet N, Cam TDT, Lam BVT, Manh HN, Gabriël S, et al. Assessing the burden of human cysticercosis in Vietnam. Trop Med Int Heal. 2013;18:352-6.

75. Taylor WRJ, Van Tran G, Nguyen TQ, Van Dang D, Nguyen VK, Nguyen CT, et al. Acute febrile myalgia in Vietnam due to trichinellosis following the consumption of raw pork. Clin Infect Dis. 2009:49:e79-83. 